\title{
Neutrophil to Lymphocyte Ratio as Biomarkers in Head and Neck Squamous Cell Carcinoma
}

\author{
Yuh Baba ${ }^{1 *}$ and Yasumasa Kato ${ }^{2}$
}

1Department of General Clinical Medicine, Ohu University, 31-1 Misumido Tomita-machi Koriyama City, Fukushima 963-8611, Japan

2Department of Oral Function and Molecular Biology, Ohu University, 31-1 Misumido Tomita-machi Koriyama City, Fukushima 963-8611, Japan

${ }^{*}$ Corresponding author: Baba Y, Department of General Clinical Medicine, Ohu University, 31-1 Misumido Tomita-machi Koriyama City, Fukushima 963-8611, Japan Tel: +81-24-932-9331 E-mail: y-baba@den.ohu-u.ac.jp

Received date: August 23, 2018; Accepted date: September 14, 2018; Published date: September 21, 2018

Citation: Baba Y, Kato Y (2018) Neutrophil to Lymphocyte Ratio as Biomarkers in Head and Neck Squamous Cell Carcinoma Biomark J. Vol.4 No. 3: 14.

Copyright: (2018 Baba Y, et al. This is an open-access article distributed under the terms of the Creative Commons Attribution License, which permits unrestricted use, distribution, and reproduction in any medium, provided the original author and source are credited.

\section{Abstract}

Head and neck squamous cell carcinoma (HNSCC) is the sixth most common neoplasm worldwide. Despite advances in treatment for HNSCC, the patient's survival remains poor. Therefore, in order to improve HNSCC patient's outcome, it is essential to explore novel biomarkers for predicting response to treatment. Herein, we state that the neutrophil to lymphocyte ratio (NLR) is a biomarker to predict the efficacy of chemoradiotherapy (CRT) in HNSCC patients and to predict the wound healing failure in head and neck reconstruction surgery. Although elevated pretreatment NLR was significantly correlated with poor response to CRT, lower preoperative NLR tended to exhibit a higher occurrence rate of postoperative wound healing failure in $\mathrm{HN}$ reconstruction surgery. Thus, in case which HNSCC patient with lower NLR has remnant tumor after CRT, salvage surgery with microsurgical reconstruction might include a higher occurrence rate of postoperative wound complications than the present. Head and neck surgeons should take care of the possibility.

\section{Keywords}

Head and neck squamous cell carcinoma; Neutrophil to lymphocyte ratio; Biomarker

\section{Introduction}

Head and neck squamous cell carcinoma (HNSCC) is the sixth most common neoplasm worldwide [1]. Despite advances in treatment, patient survival remains poor, and HNSCC is associated with a high mortality rate. Therefore, in order to improve HNSCC patient's outcome, it is essential to explore novel biomarkers for predicting response to treatment.
Tumor microenvironment is known to participate in tumor progression [2]. Inflammation is one of the microenvironment and inflammatory cells such as neutrophil and lymphocyte play an important role for tumor progression. Neutrophils are a type of inflammatory cell that secrete vascular endothelial growth factor (VEGF), chemokines, and proteases that stimulate angiogenesis, and these inflammatory cytokines may establish a tumor microenvironment and promote tumor development and progression. An increase in the number of neutrophils surrounding cancerous tissue can suppress antitumor immune response. In contrast, the number of lymphocytes reflects the activity of the lymphokine-activated killer cells, and a reduction in the number of lymphocytes results in the decreased release of cytokines which can promote apoptosis of tumor cells. Therefore, the increase of neutrophil-to-lymphocyte ratio (NLR) has been reported to predict the poor prognosis in various solid tumors such as colorectal cancer and breast cancer $[3,4]$. Herein, we state that the NLR is a biomarker to predict the efficacy of chemoradiotherapy in HNSCC patients and to predict the wound healing failure in head and neck reconstruction.

\section{Pretreatment NLR predict the chemoradiotherapy outcome in HNSCC}

Treatment with concomitant chemoradiotherapy (CRT) has emerged as a gold standard in the majority of advanced HNSCC. The presumed advantages of CRT over radical surgery are organ preservation, better functional outcome and a lower rate of acute complications. Compared to radiotherapy (RT) alone, the addition of chemotherapy (CT) delivered concomitantly with RT improves the survival of patients with advanced HNSCC with an overall $4 \%$ benefit at 5 years [5]. However, some patients treated for Stage III and IV disease does not reach to complete remission after this nonsurgical primary treatment. Therefore, it is essential to explore the biomarker to predict the efficacy of CRT. Nakashima et al. [6] reported that NLR is a potent biomarker for predicting the 
clinical response to 5-fluorouracil (5-FU)-based CRT in 124 advanced oral squamous cell carcinomas. The details of their cases as follows. All patients were treated preoperatively with CRT. RT was administered at a daily dose of 2.0 Gy five times a week for 15 days. An oral FU anti-cancer agent, S-1, was concurrently administered at a dose of 80,100 or $120 \mathrm{mg} /$ day according to each patient's body surface area for 14 days from the initiation of RT. The frequency of NLR-high $(\geq 2.4)$ patients during surgery was significantly higher among the cases who showed poor pathological response (severe destruction of tumor structure but visibly few viable tumor cells even if it works) to preoperative CRT $(P=0.038)$ [6]. Furthermore, Chandrasekara et al. [7] reported that a high NLR (>4.0) at treatment initiation is a negative predictive marker for HNSCC with cisplatin or cetuximab-based CRT. Thus, the pretreatment NLR provides significant improvement for the selection of CRT for HNSCC.

\section{Preoperative NLR predicts wound healing failure in head and neck reconstruction}

Head and neck reconstruction surgery involves a high risk of post-operative wound complication such as surgical site infections and flap necrosis (Figure 1).

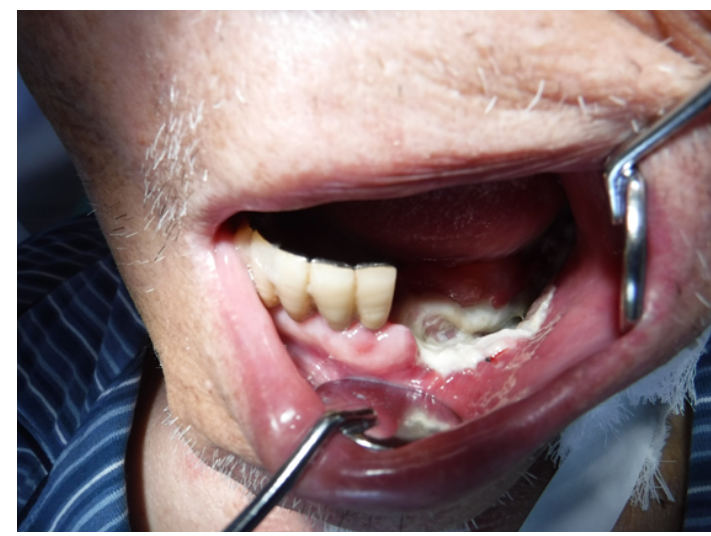

Figure 1: Flap necrosis after reconstruction for gingival cancer $(\mathrm{NLR}=2.1<3.5)$

Furthermore, such complications can require daily wound treatment and lead to prolonged hospitalization, greatly compromising the patient's quality of life. Therefore, it is necessary that we explore the predictive marker for such wound healing failure. Previously, risk factors such as a high American Society of Anesthesiologists Performance Status (ASA-PS) score and prolonged surgery time have been reported [8], recently Maruyama et al. [9] reported that the NLR differed significantly between the patients with and without post-operative wound healing failure. Lower preoperative NLR (3.5) tended to exhibit a higher occurrence rate of post-operative wound healing failure. They concluded that neutropenia and a decreased NLR may directly impair wound healing, because neutrophils migrate to the wound to prevent infection. On the contrary, Song et al. [10] reported that hypopharymgeal SCC patients with high NLR $(\geq 2.3)$ more had significantly higher incidence of wound complications $(p=0.026)$. Thus, the role of NLR as biomarker of wound healing failure seems to differ according to the subsite of HNSCC. Further work about the mechanism is needed.

\section{Conclusion}

The pretreatment NLR provides significant advantage for the selection of CRT for HNSCC. In the case by which HNSCC (excluding hypopharyngeal SCC) patient with lower NLR having remnant tumor after CRT, salvage surgery with microsurgical reconstruction might include a higher occurrence rate of postoperative wound complications than the present. Head and neck surgeons should take care of the possibility.

\section{Acknowledgments}

The present article was partly supported by a Grant-in-Aid for Scientific Research (C) 16 K11760 to Yuh Baba, Japan.

\section{Conflicts of Interest}

The authors declare there are no conflicts of interest.

\section{References}

1. Suh Y, Amelio I, Guerrero Urbano T, Tavassoli M (2014) Clinical update on cancer: Molecular oncology of head and neck cancer. Cell Death Dis 5: e1018.

2. Kato Y, Ozawa S, Miyamoto C, Maehata Y, Suzuki A, et al. (2013) Acidic extracellular microenvironment and cancer. Cancer Cell Int 13: 89.

3. Chua W, Charles KA, Baracos VE, Clarke SJ (2011) Neutrophil/ lymphocyte ratio predicts chemotherapy outcomes in patients with advanced colorectal cancer. Br J Cancer 104: 1288-1295.

4. Azab B, Bhatt VR, Phookan J, Murukutla S, Kohn N, et al. (2011) Usefulness of the neutrophil-to-lymphocyte ratio in predicting short- and long-term mortality in breast cancer patients. Ann Surg Oncol 19: 217-224.

5. Pignon JP, Bourhis J, Domenge C, Designé L (2000) Chemotherapy added to locoregional treatment for head and neck squamous-cell carcinoma:three meta-analyses of updated individual data MACH-NC collaborative Group. Meta-analysis of chemotherapy on head and neck cancer. Lancet 355: 949-955.

6. Nakashima H, Matsuoka $Y$, Yoshida R, Nagata M, Hirosue A, et al. (2016) Pre-treatment neutrophil to lymphocyte ratio predicts the chemoradiotherapy outcome and survival in patients with oral squamous cell carcinoma: A retrospective study. BMC Cancer 16:41.

7. Chandrasekara S, Davis S, Thomson P, Haydon A (2018) High neutrophil-to-lymphocyte ratio predicts poor prognosis in patients with squamous cell carcinoma of the head and neck treated with definitive chemoradiotherapy. Asia Pac J Clin Oncol.

8. Karakida K, Aoki T, Ota Y, Yamazaki H, Otsuru M, et al. (2010) Analysis of risk factors for surgical-site infections in 276 oral cancer surgeries with microvascular free-flap reconstructions at a single university hospital. J Infect Chemother 16: 334-339. 
9. Maruyama $\mathrm{Y}$, Inoue K, Mori K, Gorai K, Shimamoto R, et al. (2016) Neutrophil-lymphocyte ratio and platelet-lymphocyte ratio as predictors of wound healing failure in head and neck reconstruction. Acta Otolaryngol 137: 106-110.
10. Song Y, Liu H, Gao L, Liu X, Ma L, et al. (2015) Preoperative neutrophil-to-lymphocyte ratio as prognostic predictor for hypopharyngeal squamous cell carcinoma after radical resections. J Craniofac Surg 26: e137-140. 